\title{
Eubacterium contortum
}

National Cancer Institute

\section{Source}

National Cancer Institute. Eubacterium contortum. NCI Thesaurus. Code C86385.

A genus of aerobic, Gram-positive, rod shaped bacteria assigned to the phylum

Firmicutes. This species is nonmotile, non-spore forming, indole neg ative, reduces

neutral red, but not nitrate, does not produce hydrogen sulfide, and hydrolyzes esculin,

but not starch or hippurate. E. contortum has been isolated from human feces, vaginal swabs, and other sites of infection and nearly half of individuals with Crohn's disease have agglutinating antibodies to various strains. 